\title{
ERIKA GLASSEN
}

Zum Gedenken:

Ulrich Haarmann (22.9.1942 - 4.6.1999) 


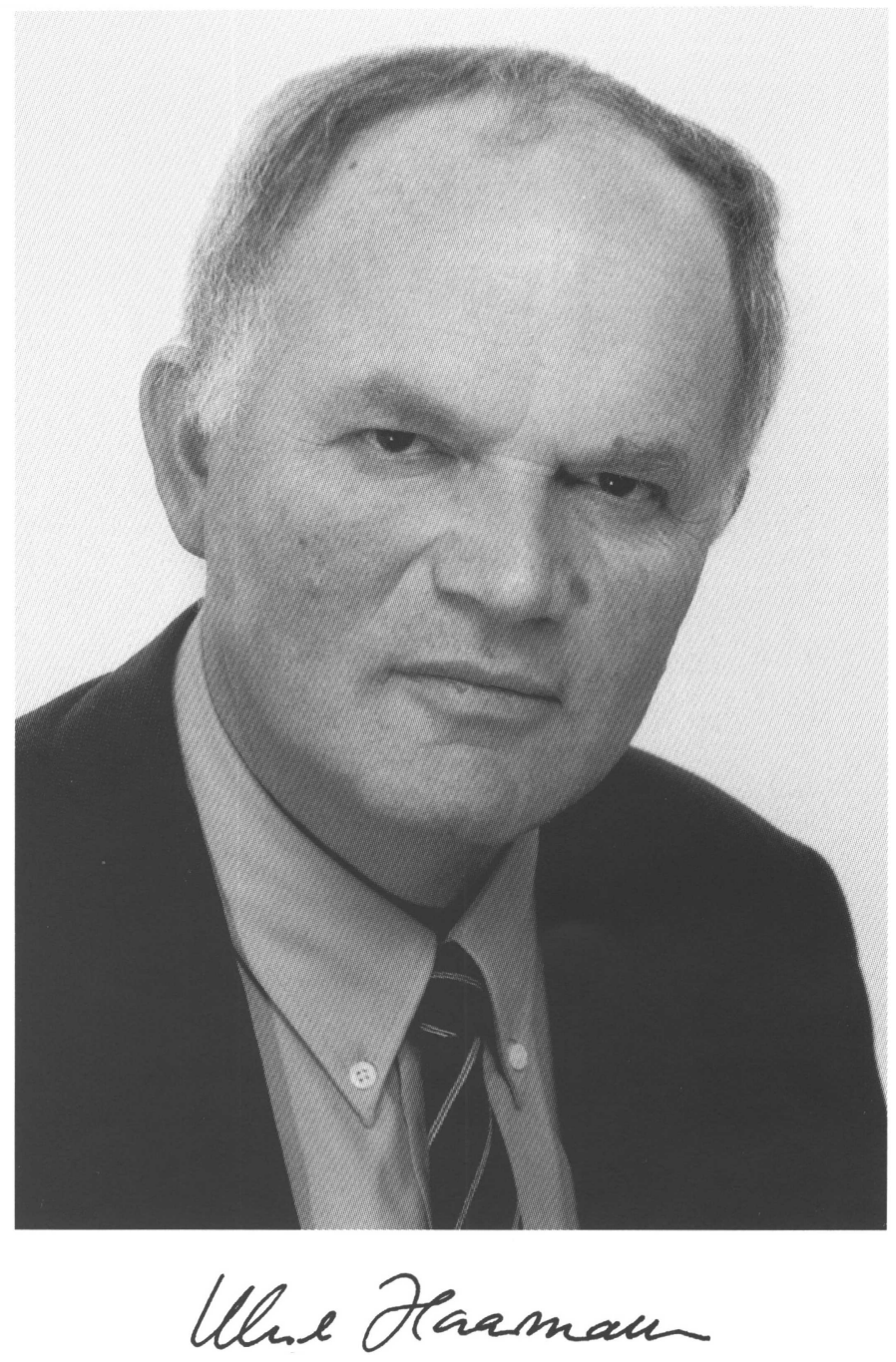




\title{
ZUM GEDENKEN: \\ ULRICH HAARMANN (22.9.1942-4.6.1999)
}

\author{
VON \\ ERIKA GLASSEN \\ Freıburg
}

\begin{abstract}
Als Ulrich Haarmann am 14. Juni 1999 auf dem Waldfriedhof in Freiburg-Littenweiler zu Grabe getragen wurde, gab ihm eine große Trauergemeinde tief erschüttert das letzte Geleit. Verwandte, Freunde, Kollegen und Schüler waren, manche von weither, angereist. Denn ungewöhnlich früh war er, von einer tückischen Krankheit mitten aus dem aktiven Leben gerissen, endgültig nach Freiburg zurückgekehrt.

Wie kaum ein anderer Fachkollege seiner Generation war Ulrich Haarmann seit seiner Studienzeit weit in der Welt herumgekommen, doch er hatte in Freiburg tiefe Wurzeln geschlagen.
\end{abstract} Sein Studienort Freiburg, nicht seine Geburtsstadt Stuttgart, war der heimatliche Hafen, in den ihn vieles immer wieder zurückzog: verwandtschaftliche, freundschaftliche und kollegiale Bande, als ruhender Pol bis zu dessen Tod 1997 unser Lehrer und Freund Hans Robert Roemer, und sicher nicht zuletzt die geliebte Schwarzwaldlandschaft und das Haus, das lange der Mittelpunkt der Familie gewesen war und das er wohl - so hofften seine Freiburger Freunde - als Emeritus wieder bewohnen wollte. Auch in den letzten Jahren, als er in Kiel und schließlich in Berlin als Ordinarius amtierte, konnte man ihm unvermutet an einer Freiburger Straßenecke begegnen. Er hatte seinen jugendlichen Habitus und seine spontane Begeisterungsfähigkeit bewahrt und erinnerte mich immer noch an die frühen gemeinsamen Jahre am Freiburger Orientalischen Seminar, die uns geprägt hatten, und an deren gewohnte Vertraulichkeit wir ohne Umschweife anknüpfen konnten.

Als wir uns kennenlernten, hatte Ulrich Haarmann schon seine 
Initiation in der Orientalistik erfahren. Zwei originelle Forscherpersönlichkeiten und verehrungswürdige Menschen waren seine ersten Lehrer und hatten in ihm den Funken der Begeisterung für das Arabische entzündet: In Freiburg der Altorientalist und Semitist Oluf Krückmann und in Amerika Rudolf Mach, unter dessen Ägide er in Princeton 1965 - als ersten akademischen Grad seinen BA in Oriental Studies über einen arabischen grammatischen Traktat aus dem 11. Jahrhundert erworben hatte. Es war zunächst die 'Arabīya, die klassische arabische Sprache als logisches, fast mathematisches System, die ihn faszinierte. Doch jenseits der systematischen Linguistik interessierte ihn bald, wie er selbst es formulierte, die arabische Sprache als Kulturobjekt in der islamischen Religion, Sprache als Bestandteil des Rechts. Er beobachtete den Widerspruch zwischen der Natürlichkeit der arabischen Sprache als eines sich wandelnden Systems und dem übernatürlichen Wundercharakter, der ihr als Sprache der göttlichen Offenbarung eigen war. Das Wort Gottes mußte als eindeutiges postuliert werden, während aus sprachlichen Unklarheiten doch Mehrdeutigkeit resultierte.

Als Haarmann aus Princeton, das er als akademisches Paradies empfunden hatte, nach Freiburg zurückkehrte, war der hochschulpolitisch engagierte Hans Robert Roemer, der 1963 auf den neugegründeten Lehrstuhl der Albert-Ludwigs-Universität für Islamkunde und Geschichte der islamischen Völker berufen worden war, dabei, das Orientalische Seminar nach seinen Vorstellungen umzugestalten und auszubauen. Roemers Forschungsinteressen galten der spätmittelalterlichen, frühneuzeitlichen Historiographie, den lange vernachlässigten ,dark ages”, in denen nach dem Mongolensturm durch turkmenisch-iranische Symbiose ungefähr in den Grenzen des vorislamischen Iran wechselnde Staatengebilde, zuletzt um 1500 die zwölferschiitische Safawidendynastie, entstanden waren. In diese Zeit gehörte auch das merkwürdige Reich der turkmenisch-tscherkessischen Mamluken in Ägypten und der Levante, das den Mongolen getrotzt und sich im Spätmittelalter als Bollwerk des arabischen sunnitischen Islams profiliert hatte. Für diese turbulente historische Phase gab es ein reiches, unerschlossenes Schrifttum, Urkunden wurden entdeckt, Hand- 
schriften von Chroniken und hagiographischen Schriften lagerten in den Bibliotheken überall auf der Welt und harrten der Bearbeitung. Aber das alles hatte nichts mit der klassischen 'Arabìya zu tun, der sich Ulrich Haarmann eigentlich widmen wollte. Doch es gelang Hans Robert Roemer, der in dem jungen hochbegabten Studenten eine förderungswürdige Kapazität witterte, ihn für ein Dissertationsthema über die Mamlukenhistoriographie zu gewinnen.

Ulrich Haarmann hat sich dieser „Dekadenzzeit" der islamischen Geschichte, in deren schriftlicher Produktion die herrschende Schicht der nicht-arabischen $a^{c} \bar{a}$ ğim vielfältige Spuren hinterlassen hat und für die auch die Erlernung des Persischen und Türkischen zur Erschließung relevanter Quellentexte unabdingbar ist, zunächst nur widerstrebend zugewandt. So erging es damals manchen Studenten, die Roemer für seine Schülerschar rekrutierte und behutsam, aber entschlossen, auf seine Wege lockte. Aber Haarmann erlag recht bald der Faszinationskraft, die die Fülle des unausgeschöpften Quellenmaterials ausstrahlte, das aus einer Zeit stammte, deren Menschen an der Schwelle zur Neuzeit stehen und uns damit schon viel näher als die Helden der islamischen Frühzeit. Er hat sich nämlich, nachdem er sich auf die Historie eingelassen hatte, nicht damit begnügt, die Ereignisgeschichte der Mamlukenperiode zu rekonstruieren, die Chronikliteratur zu sichten und in das Bezugsnetz der Gattungsgeschichte einzuordnen, den Stammbaum der Abhängigkeitsverhältnisse aufzustellen und die sprachlichen Eigenheiten des spätmittelalterlichen mit dem ägyptischen Vulgäridiom durchmischten Arabisch zu definieren usw. Das alles hat er als exzellenter Philologe keineswegs vernachlässigt, aber er hat in den Texten, mit denen er arbeitete, die er nach den zugänglichen Handschriften kritisch edierte, auch immer nach den Spuren des Individuums gesucht, das dahintersteckte. Haarmann war, so scheint mir, ein Pionier der Mentalitäten- und Rezeptionsgeschichtsforschung. Hinter den tradierten Klischees und Topoi, den Kompilationen aus oft wortwörtlich kopierten Vorlagen, ja selbst im Kalkül der ausgelassenen Stellen und zwischen den Zeilen suchte er den Menschen, der das geschrieben hatte, seine Seelenlandschaft, seine Motivationen und 
sein soziales Umfeld zu ergründen. Ihn interessierten die Adressaten und Rezipienten des Textes, die Konkurrenten und Mäzene des Verfassers.

Diese Haarmann'sche Arbeitsweise, die philologische Akribie und Detailbesessenheit im Umgang mit den Quellen und die Empathie, das sensible Gespür für ihre individuelle und soziale Komponente, hat sich bereits voll entfaltet in seiner Dissertation, die er 1969 vorlegte, erschienen 1970 unter dem Titel Quellenstudien zur frühen Mamlukenzeit (Islamkundliche Untersuchungen 1). Unter den Chronisten, deren Werke er damals untersuchte, begegnete der junge Forscher dem Mamlukensproß Ibn ad-Dawādārī (ca. 1288-1336), der ihn als origineller Geschichtsschreiber und Repräsentant der sozialen Schicht der auläd an-nās sein Leben lang beschäftigen sollte. Im Anschluß an seine Promotion verbrachte Haarmann zwei Jahre in Kairo, wo er als Wissenschaftlicher Mitarbeiter am Deutschen Archäologischen Institut den VIII. Band der Universalchronik (Kanz ad-Durar) Ibn ad-Dawādārīs kritisch edierte (Die Chronik des Ibn ad-Dawādārī, Achter Teil, Der Bericht über die Frühen Mamluken, DAI Kairo, Quellen zur Geschichte des Islamischen Ägyptens 1h, 1971).

Im Jahre 1972 habilitierte Ulrich Haarmann sich an der AlbertLudwigs-Universität in Freiburg/Br., deren Lehrkörper er (als Wissenschaftlicher Mitarbeiter, Diätendozent, apl. und C3 Professor) kontinuierlich bis 1992 angehörte, als er schließlich dem Ruf auf den Lehrstuhl für Orientalische Philologie nach Kiel folgte. Doch der Anschein der Kontinuität trügt. Sein Schreibtisch in dem Arbeitszimmer im Freiburger Orientalischen Seminar, das wir jahrelang teilten, blieb oft lange Zeit leer. Haarmanns verbindliche Art, polyglotte Begabung und akademische Gesprächskultur verschafften ihm im internationalen Wissenschaftsbetrieb viele Freunde. Immer wieder konnte er sich in Freiburg beurlauben lassen und Einladungen auf Gastprofessuren und Fellowships Folge leisten. In Ägypten (Kairo), USA (Los Angeles, Princeton und Philadelphia), Kanada (McGill University in Montréal) waren solche Stationen. Zwei Jahre lang, 1978-80, war er Direktor des von Hans Robert Roemer gegründeten Orient-Instituts der DMG in Beirut. Doch er blieb dem Freiburger Orientalischen Seminar 
treu verbunden und war eine tragende Stütze der Ära Roemer. Sicherlich hatte Ulrich Haarmann entscheidenden Anteil an dem internationalen Ruf Freiburgs als Forschungsstätte für die Geschichte und Geschichtsschreibung des islamischen Vorderen Orients im Spätmittelalter und in der frühen Neuzeit.

Haarmanns Weltläufigkeit bewahrte ihn vor einer abgekapselten, introvertierten Elfenbeinturmexistenz, und sie verhinderte, $\mathrm{da} \beta$ er sich verkrampft auf seine Hochschulkarriere fixierte, die ihm allerdings auch nicht gleichgültig war. Sein Umgang mit Kollegen und Studenten war vorbildlich. Im Vordergrund stand die unermüdliche Begeisterung für sein Fach, die er in Gesprächen und im akademischen Unterricht auf andere übertragen konnte. Die kollegiale Atmosphäre am Freiburger Orientalischen Seminar war geprägt vom produktiven Dialog, der Anteilnahme und Mitfreude am Gelingen der Arbeit der Weggefährten. Besserwisserische hämische Kritik lag ihm fern, er spendete lieber Lob und Anerkennung und ließ die Leistung der anderen gelten. Die Chance, längere Zeit in Kairo und Beirut zu leben und zu wirken und auf Kongreß- und Vortragsreisen viele andere arabische Länder kennenzulernen, half ihm nicht nur, seine Sprachbeherrschung im Arabischen zu perfektionieren, sondern auch den Blick für die islamische Moderne zu schärfen, und so gewannen Haarmanns Äußerungen über Gegenwartsprobleme auf öffentlichen Tagungen und in Zeitungsartikeln anerkannte Kompetenz. Seine Reisen und Auslandsaufenthalte hat er für seine wissenschaftliche Arbeit stets fruchtbar genutzt, ja sie kamen seinem Arbeitsstil zugute. Überall fand er eine interessante Bibliothek, wo er entlegene Literaturhinweise oder fehlende Belege entdecken, und einen Schreibtisch, an dem er seine Manuskripte ausbreiten konnte. Vor allem aber begegnete er Kollegen, mit denen er anregende wissenschaftliche und dauernde freundschaftliche Kontakte pflegen konnte.

Ulrich Haarmann arbeitete sehr lange an seinen Studien. Den spannenden Arbeitsprozeß dokumentierte er meist aufschlußreich in einer einleitenden Anmerkung. Sie wuchsen und reiften über Jahre, gewannen an Tiefenschärfe in der Argumentation und überzeugendem Belegmaterial für den Anmerkungsapparat. In 
einer ersten Phase wurden sie als Vorträge vor verschiedenen Auditorien, oft auch in mehreren sprachlichen Fassungen, vorgestellt (manchmal auch publiziert), und wichtige Quellentexte dienten in akademischen Lehrveranstaltungen als intensive Semesterlektüre. Für die abschließende Redaktion vor der Publikation kam ihm häufig ein Forschungsaufenthalt, etwa am Berliner Wissenschaftskolleg, zugute. Als typische Beispiele seien zwei seiner letzten Aufsätze genannt: „Glaubensvolk und Nation im islamischen und lateinischen Mittelalter", in Berichte und Abhandlungen, Band 2, Berlin-Brandenburgische Akademie der Wissenschaften, Berlin 1996, 161-199 und ,The Dead Ostrich, Life and Trade in Ghadames (Libya) in the Nineteenth Century" in Die Welt des Islams, Band XXXVIII, 1, 1998, 9-94.

Auch seine kritischen Editionen trugen die Spuren ihrer langwierigen Genese. Die Manuskripte und korrigierten Druckfahnen mit den üppigen, überbordenden Randnotizen in seiner klaren Handschrift, die dem Herausgeber und dem Beiruter Drucker vorgelegt wurden, sind Zeugnisse einer gelehrten Editionskultur des Zeitalters vor der Computerisierung. Ich denke an Idrīsīs Pyramidenbuch (BTS 38) und Abū Hāmid al-Qudsīs Traktat über die Segnungen, die die Türken dem Land Ägypten beschert haben (BI 37). Wenn Haarmann diese akribischen Texteditionen auch nicht als Mühsal, sondern als willkommene Exerzitien empfand, um den intensiven Umgang mit der arabischen Sprache zu pflegen, so waren es doch vor allem die Persönlichkeiten der Verfasser und ihre Themen, die ihn interessierten. Eines seiner bereits früh etablierten Arbeitsgebiete war die Beschäftigung mit den Berichten muslimischer Autoren über die ägyptischen Altertümer, besonders die Sphinx und die Pyramiden. Er wurde dazu angeregt durch seinen ersten Aufenthalt am DAI in Kairo, und da seine Frau Maria an einer Dissertation über den modernen Pharaonismus arbeitete, blieb dieses Gebiet als Gesprächsthema im Hause Haarmann immer präsent. Schon bevor er Idrīsīs Pyramidenbuch edierte, hatte er dieser Thematik, u.a. gestützt auf die Sphinxgeschichten, die Ibn ad-Dawādārī im ersten Band seiner Universalchronik erzählt, mehrere Aufsätze gewidmet, wozu auch die Erschließung des originellen und vielschichtigen Pyramidenberichts des osmani- 
schen Reisenden Evliyā Čelebī, (Turcica 8 (1976), 167-230), gehörte. Er wollte diesen Komplex weiter verfolgen, eines seiner laufenden Projekte lautete etwa: Das Babel der Zauberer, Altägypten und der mittelalterliche Islam.

Der Türken-Traktat von Abū Hāmid al-Qudsī (st. 1483) (dessen Edition er nach Subhī Labībs Tod liebevoll adoptiert hatte) brachte ihm einen Gelehrten nahe, der wie der Geschichtsschreiber Ibn ad-Dawādārī, als sensibles Individuum in einer gewissen Außenseiterposition zur Gesellschaft faßbar wird. Es gelang Ulrich Haarmann immer wieder, in seinen Quellentexten solche marginalen, empfindsamen, hypochondrischen, aufschneiderischen, umtriebigen oder nörgelnden Persönlichkeiten aufzuspüren. Dazu gehörten außer Ibn ad-Dawādārī und al-Qudsī auch der persische Jurist, Theologe und Historiker Fazlullāh b. Rūzbihān Huunğī (st. 1519) und der osmanische Gelehrte und Literat Mustafā 'Alī (st. 1600). Damit behielt Haarmann auch die islamische Ökumene im Auge. Im engagierten muslimischen Gelehrtendasein spiegelt sich besonders deutlich der Widerspruch zwischen gesetzter Norm und sozialer Wirklichkeit. Autobiographische Äußerungen und vehemente Gesellschaftskritik solcher sensiblen Naturen machen erst den Wandel im historischen Prozeß wahrnehmbar. Daher waren der Mamlukensproß Ibn ad-Dawādārī und der arabische Gelehrte al-Qudsī für ein Forschungsprojekt, das Ulrich Haarmann besonders am Herzen lag, aussagekräftige Gewährsmänner. Ihn interessierte das Spannungsverhältnis zwischen der fremdstämmigen, als Militärsklaven importierten (türkisch-tscherkessischen) politischen Machtelite der Mamluken (umara $\bar{a}^{3}$ ) und der autochthonen (arabischen) Bildungselite der Religionsgelehrten ('ulama $\vec{a}^{3}$ ). Wobei einer dritten Schicht, den in Ägypten geborenen Nachkommen der Mamluken (aulād an-nās), die nach dem herrschenden System die Privilegien ihrer Väter nicht erben durften, große Integrationsfähigkeit abverlangt wurde und eine Mittlerfunktion zukam. Gerade dieser Problematik hat Haarmann im Rahmen seiner Mamlukenforschung mehrere Studien gewidmet, zuletzt in: ,Joseph's Law The Careers and Activities of Mamluk Descendents before the Ottoman Conquest of Egypt", Ulrich Haarmann und Thomas Philipp, ed., The Mamluks in Egyptian Politics and Society. (Cam- 
bridge Studies in Islamic Civilization), Cambridge 1997. Seit 1994 gehörte Haarmann als führender Mamluken-Spezialist auch zum Herausgebergremium der an der University of Chicago erscheinenden Mamluk Studies Review.

Wenn man das unvollendete wissenschaftliche Lebenswerk Ulrich Haarmanns und seine durchgängigen Forschungsinteressen überblickt, so fällt auf, daß für ihn als Islamhistoriker seit der Beschäftigung mit der 'Arabìya die Wahrnehmung des Widerspruchs zwischen gesetzter (religiöser, juristischer) Norm und gelebter (sozialer, historischer) Realität in verschiedenen Variationen als geschichtsträchtiger Faktor fruchtbar wurde. Dazu gehörten Fragestellungen wie: Religiöses Recht und Grammatik, Auflösung und Bewahrung klassischer Formen, Hochreligion und Volksreligiosität, Dogma und geschichtliche Wirklichkeit, Glaubensvolk (umma) und Nation (watan). Solche grundsätzlichen Themen der Islamwissenschaft resümierte er auch in Aufsätzen für historisch interessierte Laien und referierte und diskutierte darüber auf Tagungen für ein breiteres Publikum.

Ulrich Haarmann war ein inspirierender Hochschullehrer, den seine Studenten in Freiburg und Kiel liebten, und er galt im interdisziplinären akademischen Rahmen als kompetenter Gesprächspartner mit komparatistischen Neigungen. Er bewährte sich als Dekan und Mitglied im Fakultätsrat und übernahm wissenschaftspolitische Funktionen, wie etwa als Projektleiter in einem Freiburger Sonderforschungsbereich, als Vertrauensdozent der Studienstiftung des Deutschen Volkes und Fachgutachter für Orientalistik der Deutschen Forschungsgemeinschaft. Er war lange Jahre Herausgeber der Reihen Bibliotheca Islamica (BI), der Quellen zur Geschichte des islamischen Ägyptens, Islamic History and Civilization, sowie zeitweise Mitherausgeber des International Journal of Middle East Studies. Besonders ehrenvoll war für ihn die Berufung zum Ordentlichen Mitglied der Academia Europaea und der BerlinBrandenburgischen Akademie der Wissenschaften.

Haarmann wirkte nur 6 Jahre (1992-1998) in Kiel. Der Freiburger war in Norddeutschland wohl noch nicht richtig seßhaft geworden. In einer Zeit der knappen öffentlichen Geldmittel konnte man seine Visionen an einem Universitätsseminar nicht so leicht 
verwirklichen wie vormals während Roemers Aufbauphase in Freiburg. Daher war für Ulrich Haarmann der Ruf nach Berlin als C4Professor für Islamwissenschaft an die FU (Lehre) und auf den Posten des Direktors am Zentrum Moderner Orient (Forschung und Wissenschaftsmanagement) verlockend. Er hatte Lust dazu, sich nun vermehrt modernen Themen zuzuwenden, sozusagen amtshalber. Hier hatte er erstmals einen großen Mitarbeiterstab zur Verfügung, mit dem er gemeinsame Forschungsprojekte erarbeiten und sich am Aufbau von Netzwerken der deutschen und internationalen Wissenschaftsinstitutionen beteiligten konnte. Seine internationalen Kontakte und sein öffentliches Ansehen, seine liebenswürdige, kommunikative Art sowie die Fähigkeit, mit echter Begeisterung für das Fach und spürbarer wissenschaftlicher Neugier Studenten und Kollegen zu motivieren und ermutigen, prädestinierten ihn für sein neues Amt, das er nur 14 Monate vor seinem Tod mit so viel Elan angetreten hatte. Die deutsche Islamwissenschaft hat mit Ulrich Haarmann den allzu frühen Verlust eines ihrer fähigsten und anerkanntesten Vertreter zu beklagen und wird ihm ihr ehrendes Andenken bewahren. 